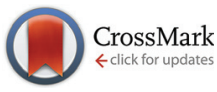

Cite this: Org. Biomol. Chem., 2016, 14, 3695

Received 24th February 2016, Accepted 17th March 2016

DOI: $10.1039 / c 6 o b 00430 j$

www.rsc.org/obc

\section{A surprising switch in absolute configuration of anti-inflammatory macrolactones $\uparrow$}

\author{
Johannes Tauber, ${ }^{a}$ Markus Rohr, ${ }^{\mathrm{b}}$ Thorsten Walter, ${ }^{\mathrm{b}}$ Dieter Schollmeyer, ${ }^{\mathrm{a}}$ \\ Karin Rahn-Hotze, ${ }^{c}$ Gerhard Erkel ${ }^{b}$ and Till Opatz ${ }^{* a}$
}

\begin{abstract}
Oxacyclododecindione-type macrolactones exhibit highly potent anti-inflammatory activities even at nanomolar concentration. After the determination of the relative configuration of the stereocenters at C14 and C15 by total synthesis of 4-dechloro-14-deoxyoxacyclododecindione and 14-deoxyoxacyclododecindione, the absolute configuration has now been assigned by $\mathrm{X}$-ray crystallography. Surprisingly, the absolute configuration is $(14 S, 15 R)$ which differs for C15 from that of the well-known derivatives of (S)-curvularin. The biological activities of both enantiomers of 14-deoxyoxacyclododecindione, obtained by racemic synthesis and optical resolution, were investigated and the ring conformation of the natural product was compared to that of $(S)$-curvularin and $(R)$ dehydrocurvularin.
\end{abstract}

\section{Introduction}

The 12-membered macrolactones $(S)$-curvularin $((-)-\mathbf{1}),{ }^{1}(S)$ 10,11-dehydrocurvularin ((-)-2), ${ }^{1}$ 4-dechloro-14-deoxyoxacyclododecindione (3), ${ }^{2}$ 14-deoxyoxacyclododecindione $(4),{ }^{2}$ and oxacyclododecindione $(5)^{3}$ were all isolated as fungal secondary metabolites (Fig. 1). While the absolute configurations of $(S)$-curvularin ((-)-1) and (S)-10,11-dehydrocurvularin ((-)-2) were proven by total synthesis, ${ }^{4-11}$ it could not yet be determined for the macrolactones 3-5. It should be noted that the relative configuration of the two adjacent stereocenters was assigned as $\left(14 R^{*}, 15 S^{*}\right)$ by racemic total synthesis of 4-dechloro-14-deoxyoxacyclododecindione (3) and 14-deoxyoxacyclododecindione (4). ${ }^{12,13}$ Due to their structural analogy to the macrolactones $\mathbf{1}$ and $\mathbf{2}$ the absolute configuration was

\footnotetext{
${ }^{a}$ Institute of Organic Chemistry, Johannes Gutenberg-University, Duesbergweg 10-14, 55128 Mainz, Germany.E-mail: opatz@uni-mainz.de

${ }^{b}$ Department of Molecular Biotechnology \& Systems Biology, University of Kaiserslautern, Erwin-Schrödinger Str. 70, Building 70, 67663 Kaiserslautern, Germany

${ }^{c} R \& D$ LGCR/Chemistry FF, Sanofi GmbH, D-65926 Frankfurt am Main, Germany $\dagger$ Electronic supplementary information (ESI) available. CCDC 1454207. For ESI and crystallographic data in CIF or other electronic format see DOI: 10.1039/ c6ob00430j
}

assumed to be $(14 R, 15 S) .{ }^{12-15}$ While (-)-1 and (-)-2 show biological activities in the micromolar range, the secondary metabolites 3-5 exhibit anti-inflammatory and anti-fibrotic activities in cell culture experiments at nanomolar concentrations. ${ }^{2,3}$ Furthermore, these lactones were successfully used in an in vivo mouse model of systemic lupus erythematosus (SLE). ${ }^{16}$ They may thus serve as lead structures for therapeutics against chronic inflammatory and/or fibrotic diseases like asthma, rheumatoid arthritis, SLE or cancer. ${ }^{2,16}$

\section{Results and discussion}

In our previous report on the racemic total synthesis of 4-dechloro-14-deoxyoxacyclododecindione $(( \pm)-3)$ and 14-deoxyoxacyclododecindione $(( \pm)-4)$, we found that synthetic $( \pm)-\mathbf{4}$ was more active on a IL-4/Stat6-dependent and a TGF- $\beta / \mathrm{Smad} 2 / 3$ dependent transcriptional luciferase reporter in transiently transfected HepG2 cells, than the isolated natural product $(+)-4 .^{12,13}$ There are two possibilities to explain this result; the isolated material had a lower degree of purity, or the unnatural enantiomer $(-)-4$ is more active than the naturally occurring enantiomer $(+)-4$. To investigate this issue and to elucidate the absolute configuration of 14-deoxyoxacyclododecindione $((+)-4)$, a separation of racemic ( \pm )-4 by chiral, preparative HPLC was performed.

Comparison of the optical rotations of the pure synthetic enantiomers of $4\left([\alpha]_{\mathrm{D}}^{26}=+23^{\circ}(c=0.57 \mathrm{~g}\right.$ per $100 \mathrm{~mL}, \mathrm{MeOH})$ and $[\alpha]_{\mathrm{D}}^{26}=-21^{\circ}(c=0.55 \mathrm{~g}$ per $\left.100 \mathrm{~mL}, \mathrm{MeOH})\right)$ with the respective value of the natural product $\left([\alpha]_{\mathrm{D}}^{22}=+9.6^{\circ}(c=0.34 \mathrm{~g}\right.$ per $100 \mathrm{~mL}$, methanol- $\left.\mathrm{d}_{4}\right)$ ) allowed the assignment of the naturally occurring isomer as the dextrorotatory enantiomer. ${ }^{2}$ Crystallization and subsequent X-ray crystallography of this enantiomer using anomalous dispersion at the chlorine substituent revealed that its absolute configuration is $(14 S, 15 R)$. This is in contrast to the assumed configuration and is rather surprising because the structurally related macrolactones (-)-1 and $(-)-2$ have the $(S)$-configurations at C15. ${ }^{17-19}$ A comparison of the crystal structures of $(+)-4$ with $(-)-\mathbf{1}^{8,20}$ shows that the carbonyl group in both cases is perpendicular to the aromatic 
<smiles>C[C@H]1CCCCCC(=O)c2c(O)cc(O)cc2CC(=O)O1</smiles>

(S)-curvularin<smiles>COC(C)CC/C=C/C(=O)c1c(O)cc(O)cc1CC(=O)O</smiles>

(S)-10,11-dehydrocurvularin
$\mathrm{HO}$<smiles>CC(=CCCC(C)(C)C)C(=O)c1c(O)cccc1CC(=O)O</smiles>

4-dechloro-14-deoxyoxacyclododecindione<smiles>CC(=CCCC(C)(C)C(C)OC(=O)Cc1c(C)c(O)cc(O)c1Cl)C(=O)O</smiles>

14-deoxyoxacyclododecindione

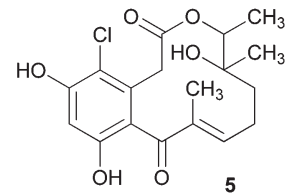

oxacyclododecindione

increasing anti-inflammatory potency

Fig. 1 Structures of the secondary metabolites 1-5.
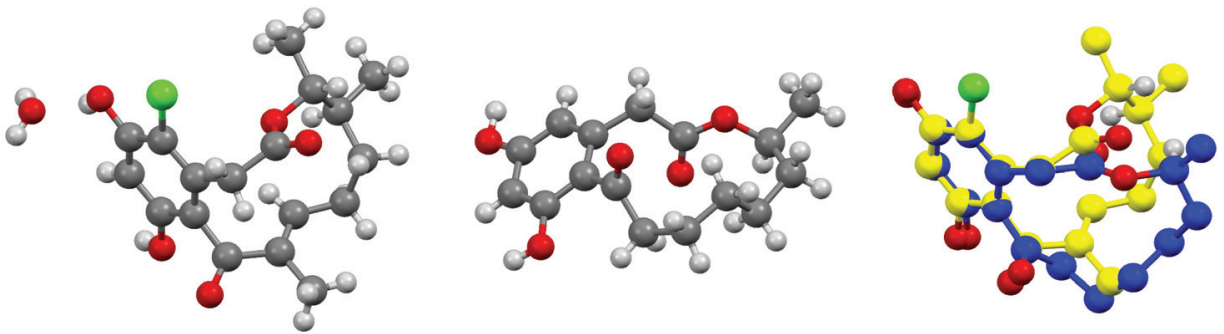

Fig. 2 Crystal structure of (14S,15R)-14-deoxyoxacyclododecindione ((+)-4) (left: hydrate), (15S)-curvularin ((-)-1) (center) and overlay of (+)-4 and (-)-1 (right, blue: (+)-4, yellow: (-)-1).

system and that the ester group adopts the trans-configuration (Fig. 2). However, the conformation of the 12-membered ring in (+)-4 differs considerably and is even less similar to that of $(R)$-curvularin $(+)-1^{20,21}$ (Fig. 3). Thus, the double bond

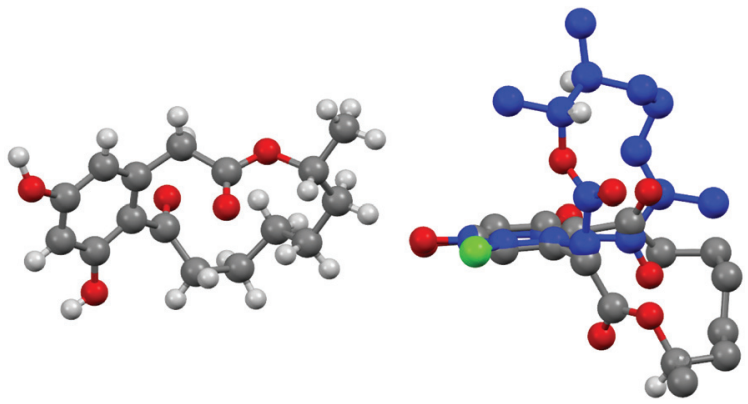

Fig. 3 X-ray structures of (15R)-curvularin ((+)-1) (left) and overlay with (+)-4 (right, blue: (+)-4, grey: (15R)-curvularin (+)-1). between C10-C11 appears to have a significant effect on the ring conformation. Therefore, the crystal structure of (+)-4 was compared with the structure of $(15 S)$-dehydrocurvularin $(-)-2^{22}$ and its enantiomer $(+)-2,{ }^{19}$ the conformations of which were previously discussed by Dai et al. and differ from each other due to the presence of solvent molecules in the crystal (Fig. 4). ${ }^{19}$ The crystal structures of (+)-4 and (+)-2 reveal a highly similar spatial orientation of both 12-membered rings and demonstrate that the ring conformation is largely governed by the unsaturation.

The reason for the inconsistency in the orientation of the methyl group at C15 between the oxacyclododecindione and the curvularin series is unclear and worthwhile of investigation. Given the close structural relationship of lactone 4 and oxacyclododecindione (5), it might be speculated that the latter natural products could have $(14 R, 15 R)$-configuration if 4 is its direct biogenetic precursor but an independent analysis is absolutely required in view of the nonuniform stereochemistry within this series.
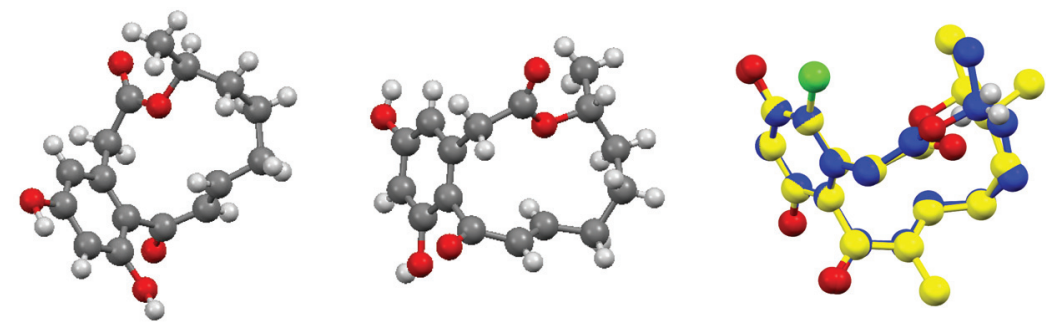

Fig. 4 X-ray structure of (15S)-dehydrocurvularin (-)-2 (left), (15R)-dehydrocurvularin (+)-2 (center), and overlay of $(+)-4$ and (+)-2 (right, blue: (+)-2, yellow: (+)-4). 
Table 1 Effect of 14-deoxyoxacyclododecindione (4) in two relevant reporter gene assays

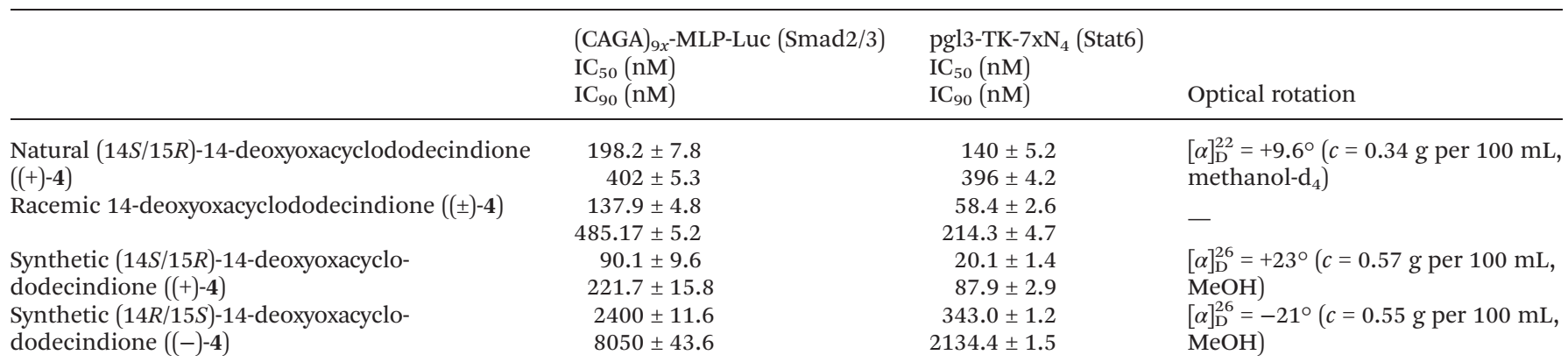

HepG2 cells were transiently transfected with the indicated reporter gene construct and the constitutively active pRL-EF1 $\alpha$ reporter gene and stimulated with $5 \mathrm{ng} \mathrm{mL}{ }^{-1}$ TGF- $\beta$ or $5 \mathrm{ng} \mathrm{mL} \mathrm{LL}^{-1} \mathrm{IL}$ with or without test compounds for $16 \mathrm{~h}$ as described in Materials and methods.

\section{Biological evaluation}

Both enantiomers of 14-deoxyoxacyclododecindione (4) were tested for their inhibitory activity on IL-4 inducible Stat6dependent and TGF- $\beta$ inducible Smad $2 / 3$ dependent transcriptional luciferase reporter assays using transiently transfected HepG2 cells (Table 1). These two reporter gene assays were selected since the JAK/STAT- and the TGF- $\beta$ signal transduction pathway play key roles in inflammatory and fibrotic diseases.

The synthetic, naturally occurring enantiomer (+)-4 at concentrations of $\mathrm{IC}_{50}=90 \mathrm{nM}(\mathrm{Smad})$ and $\mathrm{IC}_{50}=20 \mathrm{nM}$ (Stat6) is around 27- and 17-fold more active than its synthetic antipode (-)-4 (2400 nM and $343 \mathrm{nM}$, respectively, as measured for $\mathrm{IC}_{50}$ and supported by the $\mathrm{IC}_{90}$ data, see Table 1 ). Thus, the second hypothesis (see above) was disproved and the higher activity of synthetic (+)-4 compared to the isolated natural product (198 nM and $140 \mathrm{nM}$ ) appears to originate from a higher degree of purity which is consistent with the higher optical rotation of synthetic (+)-4.

\section{Conclusion}

In summary, the absolute configuration of 14-deoxyoxacyclododecindione $((+)-\mathbf{4})$ was assigned by optical resolution of $( \pm)-\mathbf{4}$ using chiral preparative HPLC and X-ray crystallography. Surprisingly, the absolute configuration is $(14 S / 15 R)$. Furthermore, the biological activities of both enantiomers were investigated and illustrated that the synthetic naturally occurring (+)-4 is around 20-fold more active than synthetic (-)-4.

\section{Experimental section}

\section{Material and methods}

Analytical, chiral HPLC was performed on a Daicel chiralpak

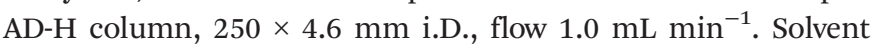
system heptanes : ethanol : methanol $5: 1: 1 . R_{\mathrm{t}}((+)-4)=6.2 \mathrm{~min}$, $R_{\mathrm{t}}((-)-4)=4.5 \mathrm{~min}$. Preparative, chiral HPLC was performed on

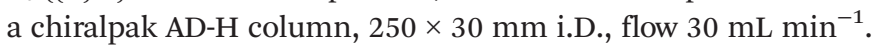
Solvent system heptane : ethanol : methanol $5: 1: 1$.
The polarimeter values were measured with a Perkin-Elmer 241 polarimeter at $546 \mathrm{~nm}$ and $578 \mathrm{~nm}$. The data were extrapolated to a wavelength of $\lambda=589 \mathrm{~nm}$ by using the Drude equation. $^{23}$

Crystallization was performed by the solvent diffusion method using ethyl acetate and petroleum ether.

X-ray crystallography of (+)-4 was performed on a STOE IPDS $2 \mathrm{~T}$ with $\mathrm{Mo}-\mathrm{K}_{\alpha}$ radiation (graphite monochromator).

CCDC 1454207 contains the supplementary crystallographic data for (14S/15R)-14-deoxyoxacyclododecindione (+)-4.

\section{Reporter gene assays}

HepG2 cells (DSMZ ACC 180) were cultured in DMEM medium supplemented with $10 \%$ fetal calf serum (FCS) and $65 \mu \mathrm{g}$ per $\mathrm{mL}$ penicillin $\mathrm{G}$ and $100 \mu \mathrm{g}$ per $\mathrm{mL}$ streptomycin sulfate in a humidified atmosphere. The Stat6-dependent transcriptional activation assay was performed by transfection of HepG2 cells with the Stat6-driven reporter plasmid pGL3-TK-7 $\mathrm{xN}_{4}$ which contains the herpes simplex virus thymidine kinase promoter under the control of 7 copies of the palindromic sequence TTC $(\mathrm{N})_{4}$ GAA together with a Stat6 expression plasmid (TOPOStat6). ${ }^{3}$ The TGF- $\beta$ inducible $\operatorname{Smad} 2 / 3$ dependent reporter plasmid (AGCCAGACA) ${ }_{9}$ MLP-Luc was a kind gift from Prof. Dr S. Dooley (University of Mannheim, Germany) and was described previously. ${ }^{24}$ The control reporter vector pRL-EF1 $\alpha$ for data normalization was purchased from Promega (DualLuciferase-Reporter-Assay). Luciferase-based reporter gene expression was thereby normalized for transfection variability and cytotoxicity against renilla expression of the constitutively active control vector (pRL-EF1 $\alpha$ ) assayed in the same sample. Transfection of HepG2 cells were performed by electroporation (Bio-Rad, Gene-Pulser) of $1 \times 10^{7}$ cells per $\mathrm{mL}$ DMEM together with of the indicated constructs $(50 \mu \mathrm{g})$ and the internal control pRL-EF1 $\alpha$ vector at $500 \mathrm{~V} \mathrm{~cm}^{-1}$. After electroporation the cells were seeded at $2 \times 10^{5}$ cells per $\mathrm{mL}$ and allowed to recover for $16 \mathrm{~h}$. For induction of reporter gene expression, the cells were treated either with $5 \mathrm{ng} \mathrm{mL}{ }^{-1}$ TGF- $\beta$ or $5 \mathrm{ng} \mathrm{mL}^{-1}$ IL-4 with or without test compounds in DMEM containing $5 \%$ FCS. Luciferase activity was measured $16 \mathrm{~h}$ after induction using the luciferase assay system (Promega, Mannheim, 
Germany) according to the manufacturer's instructions with a luminometer.

\section{Acknowledgements}

J. T. is grateful to the Fonds der Chemischen Industrie for a PhD-fellowship. This work was supported by the Rhineland Palatinate Center for Natural Product Research and the Zeiss foundation.

\section{References}

1 O. C. Musgrave, J. Chem. Soc., 1956, 4301-4305.

2 J. Richter, L. P. Sandjo, J. C. Liermann, T. Opatz and G. Erkel, Bioorg. Med. Chem., 2015, 23, 556-563.

3 G. Erkel, H. Belahmer, A. Serwe, T. Anke, H. Kunz, H. Kolshorn, J. Liermann and T. Opatz, J. Antibiot., 2008, 61, 285-290.

4 H. Gerlach, Helv. Chim. Acta, 1977, 60, 3039-3044.

5 F. Bracher and B. Schulte, Nat. Prod. Lett., 1995, 7, 65-68.

6 F. Bracher and B. Schulte, Liebigs Ann./Recl., 1997, 1997, 1979-1982.

7 D. De Joarder and M. P. Jennings, Eur. J. Org. Chem., 2015, 3303-3313.

8 S. Elzner, D. Schmidt, D. Schollmeyer, G. Erkel, T. Anke, H. Kleinert, U. Förstermann and H. Kunz, ChemMedChem, 2008, 3, 924-939.

9 D. K. Mohapatra, H. Rahaman, R. Pal and M. K. Gurjar, Synlett, 2008, 1801-1804.

10 P. M. Tadross, S. C. Virgil and B. M. Stoltz, Org. Lett., 2010, 12, 1612-1614.
11 P. Persich, J. Llaveria, R. Lhermet, T. de Haro, R. Stade, A. Kondoh and A. Fürstner, Chem. - Eur. J., 2013, 19, 13047-13058.

12 J. Tauber, M. Rohr, T. Walter, G. Erkel and T. Opatz, Org. Biomol. Chem., 2015, 13, 7813-7821.

13 J. Tauber, M. Rohr, T. Walter, G. Erkel and T. Opatz, Org. Biomol. Chem., 2015, 13, 8945-8946.

14 J. Tauber, K. Rudolph, M. Rohr, G. Erkel and T. Opatz, Eur. J. Org. Chem., 2015, 3587-3608.

15 J. Tauber, K. Rudolph, M. Rohr, G. Erkel and T. Opatz, Eur. J. Org. Chem., 2015, 5671-5671.

16 J. Henke, G. Erkel, C. Brochhausen, H. Kleinert, A. Schwarting, J. Menke and A. Pautz, Kidney Int., 2014, 86, 780-789.

17 J. Xu, C. S. Jiang, Z. L. Zhang, W. Q. Ma and Y. W. Guo, Acta Pharmacol. Sin., 2014, 35, 316-330.

18 W. Shen, H. Mao, Q. Huang and J. Dong, Eur. J. Med. Chem., 2015, 97, 747-777.

19 For rare cases of curvularin-type macrolactones with $15 R$ configuration, see: J. Dai, K. Krohn, U. Flörke, G. Pescitelli, G. Kerti, T. Papp, K. E. Kövér, A. C. Bényei, S. Draeger, B. Schulz and T. Kurtán, Eur. J. Org. Chem., 2010, 69286937.

20 S. Elzner, $P h D$ thesis, Johannes Gutenberg-Universität, Mainz, 2005.

21 S. Elzner, H. Kunz and D. Schollmeyer, private communication, 2016, CCDC 1454220.

22 F. Almassi, E. L. Ghisalberti, B. W. Skelton and A. H. White, Aust. J. Chem., 1994, 47, 1193-1197.

23 G. Lippke and H. Thaler, Starch/Staerke, 1970, 22, 344351.

24 S. Dennler, S. Itoh, D. Vivien, P. ten Dijke, S. Huet and J. M. Gauthier, Embo J., 1998, 17, 3091-3100. 